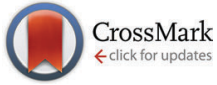

Cite this: Chem. Commun., 2014, 50, 10094

Received 28th May 2014,

Accepted 4th July 2014

DOI: $10.1039 /$ c4cc04092a

www.rsc.org/chemcomm

\section{Ligand-controlled divergent formation of alkenyl- or allylboronates catalyzed by Pd, and synthetic applications $\dagger$}

\author{
Alicia Martos-Redruejo, Ruth López-Durán, Elena Buñuel and Diego J. Cárdenas*
}

The use of different ligands allows the preparation of either allyl- or alkenylboronates by Pd-catalyzed borylation of allylic carbonates containing alkyne groups. Unprecedented borylative cyclisation to alkenylboronates takes place with $\mathrm{PCy}_{3}$. The difficult dissociation of NHC ligands allows borylation of carbonates in the presence of alkynes. Oxidation, regioselective Suzuki coupling, as well as Au-catalyzed cycloisomerisation of boronates illustrate the potential synthetic applications of these reactions.

Boronates are important synthetic reagents. They are stable and compatible with most functional groups and are environmentally friendly, tolerating oxygen and water. A wide variety of boronates can be used as nucleophilic partners in Suzuki cross-coupling reactions and allylboronates are reactive towards aldehydes and imines, just to cite the most common uses of these compounds. ${ }^{1}$ For this reason, the development of methods for the preparation of boronates, avoiding the use of highly nucleophilic and basic reagents is of main interest. On the other hand, enynes constitute versatile starting materials for the formation of carbo and heterocycles, ${ }^{2}$ skeletal rearrangements, ${ }^{3}$ and alkoxy-cyclisation, ${ }^{4}$ among other reactions. During the last few years, Pd-catalyzed borylative cyclisation reactions of polyunsaturated species have been developed by our group ${ }^{5-9}$ and Bäckvall's group, ${ }^{10}$ with the aim of preparing cyclized boronates. Our seminal work described an unprecedented preparation of homoallylic boronates starting with 1,6-enynes, in which esters substituting the allylic position were tolerated (Scheme 1, top)., ${ }^{5,11}$ In our previous studies, addition of ligands precluded the incorporation of the boron substituent. Now, we have found conditions in which the choice of the appropriate ligand allows control of the borylation of alkyne-containing allylcarbonates, affording simple Miyaura coupling to allylboronates, or unprecedented formation of alkenylboronates by borylative cyclisation, a method which is

Departamento de Química Orgánica, Facultad de Ciencias, Universidad Autónoma de Madrid, Campus de Cantoblanco, 28049-Madrid, Spain. E-mail: diego.cardenas@uam.es; Fax: +34 914973966; Tel: +34 914974358 $\dagger$ Electronic supplementary information (ESI) available: Experimental procedures, data and computational details. CCDC 1005655-1005657. For ESI and crystallographic data in CIF or other electronic format see DOI: $10.1039 / \mathrm{c} 4 \mathrm{cc} 04092 \mathrm{a}$
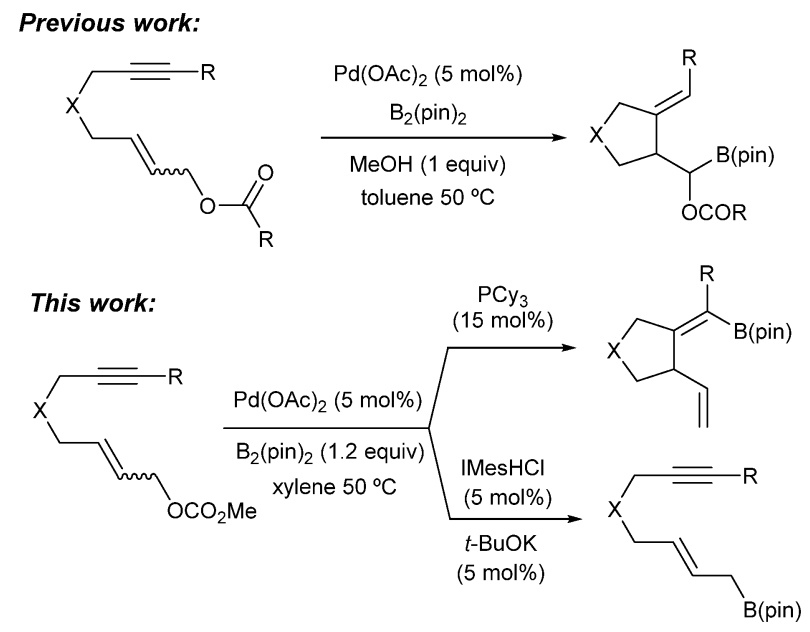

Scheme $1 \mathrm{Pd}$-catalyzed borylation reactions of substituted 1,6-enynes.

complementary to alkyne hydroboration and the usual reactions of strongly basic $\mathrm{Li}$ and $\mathrm{Mg}$ nucleophiles with $\mathrm{B}(\mathrm{OMe})_{3}$.

Thus, we started our study by reacting enynes containing an allylic carbonate group (1). In our previous work mentioned above, ${ }^{5}$ we reported that reactions of acetates or other ester derivatives, under ligandless conditions, took place without activation of the C-O bond, leading to homoallylic boronates containing the unreacted ester group (Scheme 1, top). Reactions in the presence of phosphines led to simple cycloisomerisation without incorporation of the boryl group.

In contrast, reaction of carbonates $\mathbf{1 a - 1}$ with $\mathrm{B}_{2}(\mathrm{pin})_{2}$ in the presence of $\mathrm{Pd}(\mathrm{OAc})_{2}$ and the IMes ligand (formed in situ by deprotonation of the imidazolium chloride with $t$-BuOK) in xylene, afforded the corresponding allylboronates (Table 1). The reaction is fast (less than $30 \mathrm{~min}$ ) and takes place under optimum conditions $\left(50{ }^{\circ} \mathrm{C}\right)$ in the absence of added base, as usual for carbonates. A variety of starting compounds showing different tethering groups between the alkene and the alkyne (malonate and NTs) were used. The allylboronate product always shows an $(E)$ configuration on the alkene, regardless the configuration of the $\mathrm{C}-\mathrm{C}$ double bond on the 
Table 1 Pd-catalyzed formation of allylboronates
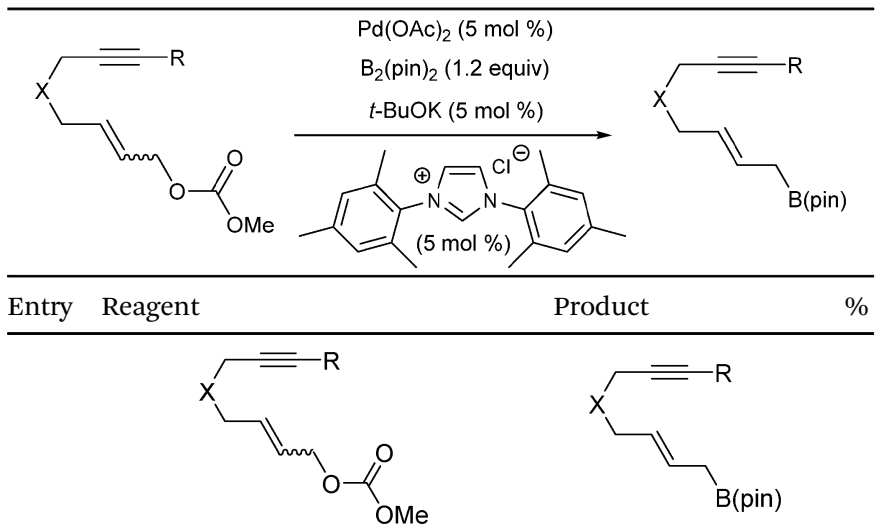

Product

$\%$

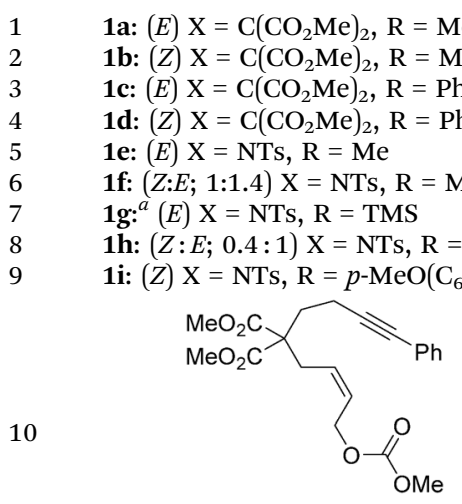

$1 \mathrm{j}$

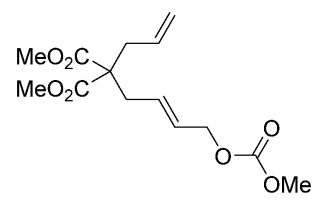

$1 \mathrm{k}$

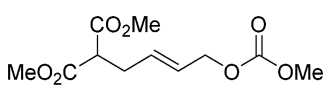

11

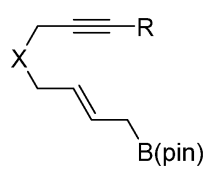

\begin{abstract}
2ab
2ab

2cd

2cd

2ef

$2 \mathrm{ef}$

2gh

$2 \mathrm{gh}$

$2 \mathbf{i}$
\end{abstract}

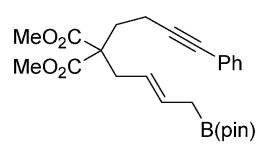

37

2j

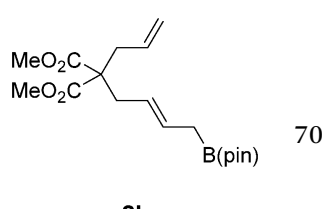

2k

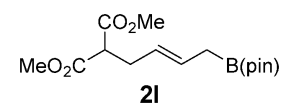

59

Reaction conditions: xylene mixture, $50{ }^{\circ} \mathrm{C}, 25 \mathrm{~min} .{ }^{a}$ Contains $9 \%$ of $Z$ isomer.

starting material (see Table 1: entries 1-6). Terminal alkynes gave complex reaction mixtures, probably for the occurrence of other reactions, but the reaction could be extended to alkyl, aryl and TMS substituted alkynes. Phenyl substituted alkynes provided lower yields (entries 3 and 4), but $p$-MeO-phenyl derivative $1 \mathbf{i}$ gave the corresponding boronate $2 \mathbf{i}$ in high yield (entry 9). The presence of the triple bond is compatible with these reaction conditions, the alkyne remaining unreacted. Finally, compounds $\mathbf{1 k}$ and $\mathbf{1 l}$ also afforded the desired products, as expected for these simpler reagents. In summary, this constitutes a Miyaura reaction for the formation of allylboronates under optimum conditions and in the presence of internal alkynes. $^{12}$

Interestingly, when the reaction was performed in the presence of $\mathrm{Pd}(\mathrm{OAc})_{2}$ and $\mathrm{PCy}_{3}$ as a ligand, a completely different outcome was observed. A novel borylative cyclisation led to alkenylboronates $\mathbf{3}$,
Table 2 Pd-catalyzed borylative cyclisation to alkenylboronates

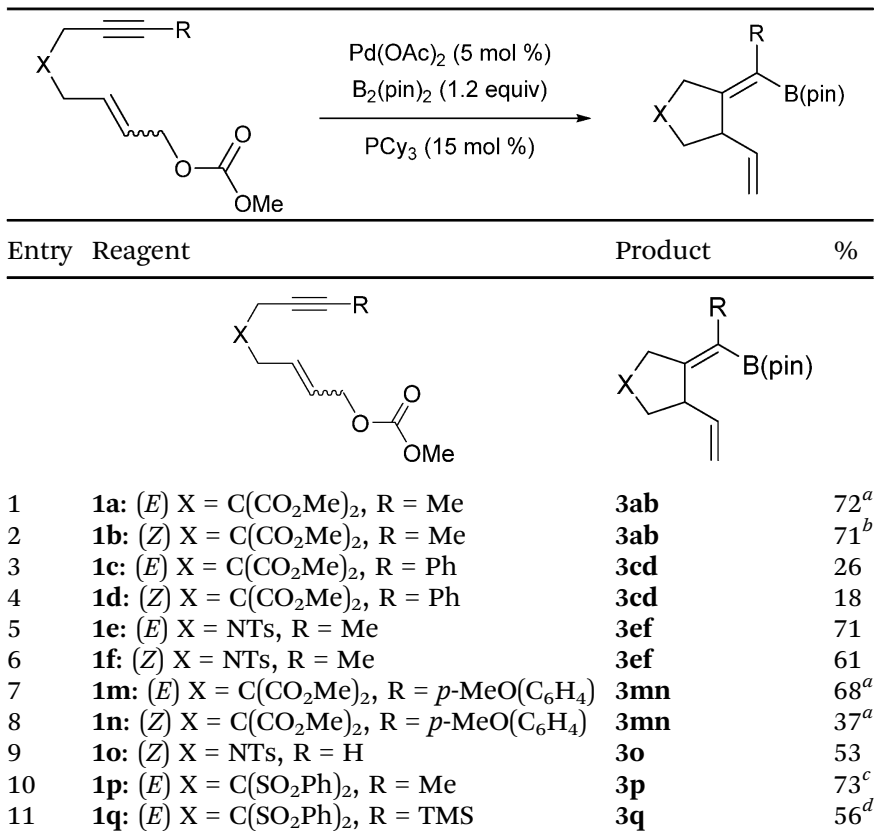

Reaction conditions (unless otherwise stated): xylene mixture, $50{ }^{\circ} \mathrm{C}$, $8 \mathrm{~h}^{a}{ }^{a}$ Reaction performed in 1,4-dioxane. ${ }^{b}$ 1,4-Dioxane, $110^{\circ} \mathrm{C}, 2.5 \mathrm{~h}$. ${ }^{c} \mathrm{Pd}(\mathrm{OAc})_{2}, 15 \mathrm{~mol} \%$ (65\% with $\left.5 \mathrm{~mol} \%\right) .{ }^{d} 130{ }^{\circ} \mathrm{C}, 40 \mathrm{~min}$.

isomers of the above mentioned allylboronates (Table 2). The reaction leads to the formation of one $\mathrm{C}-\mathrm{C}$ and one $\mathrm{C}-\mathrm{B}$ bond in a single operation with formation of five-membered carbo- or heterocycles. ${ }^{13}$ This process is stereoselective, affording a single configurational isomer of the alkenylboronate. Crystal structures of 3mn (Fig. 1) and 3p (ESI $\dagger$ ) confirmed the stereochemistry of the new $\mathrm{C}-\mathrm{C}$ double bond. Again, the reaction shows a broad scope, and more interestingly, terminal alkyne 10 also gave the corresponding product. Bis(phenylsulfonyl)methane derivatives also afforded the expected compounds in good yields, in contrast to the observation under the conditions provided in Table 1: allylboronates from bis(sulfonyl) were detected in low yields in mixtures that could not be separated. A TMS-substituted product (3q) could be obtained again, in spite of the presence of alkoxide in the reaction medium.

A mechanistic rationale for the formation of allyl- or alkenylboronates is depicted in Scheme 2. We propose reduction of the precatalyst to $\operatorname{Pd}(0)$ followed by oxidative addition of the allyl carbonate and decarboxylation to give allyl complex A. If a fast transmetalation process takes place, boryl-Pd intermediate $\mathbf{B}$ would evolve to allylboronate by regioselective $\mathrm{C}-\mathrm{B}$ reductive elimination, and a catalytically active $\operatorname{Pd}(0)$ species. Alternatively, coordination of the alkyne of complex $\mathbf{A}$ to give $\mathbf{C}$, followed by carbometallation
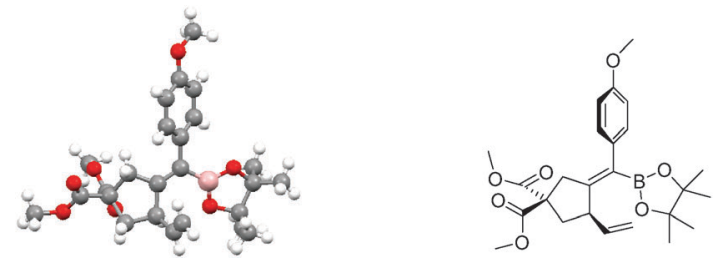

Fig. 1 Crystal structure of $3 \mathrm{mn}$. 


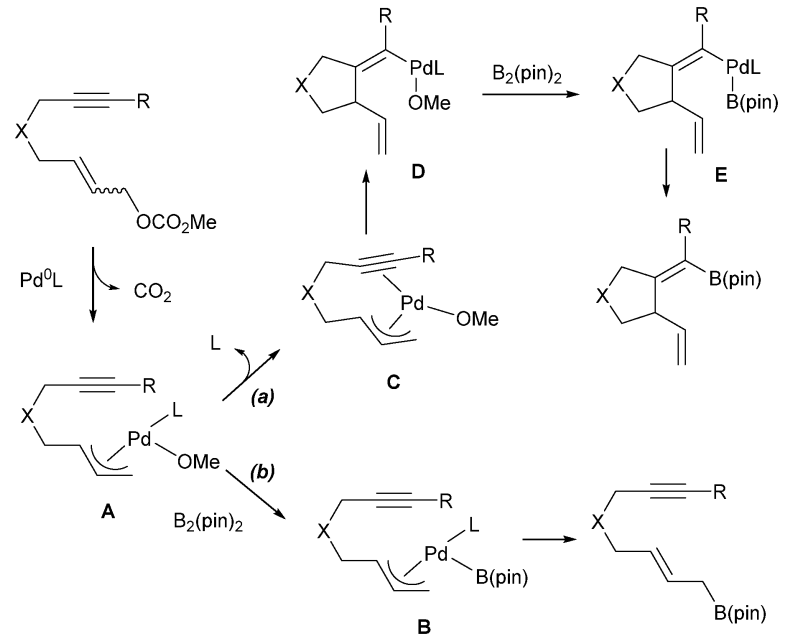

Scheme 2 Proposed reaction pathway for both reactions.

would provide alkenyl-Pd derivative D. Subsequent transmetalation leading to $\mathbf{E}$ and reductive elimination would generate the alkenylboronates. Noteworthily, hindered trialkylphosphines and NHC ligands usually confer similar properties to Pd complexes. Thus, activation of aryl chlorides, and inhibition of $\beta$-hydrogen elimination of alkyl-Pd intermediates in coupling reactions can be achieved with both kinds of ligands. ${ }^{14,15}$

We hypothesised that the reason for the differential behavior observed for these ligands in the formation of boronates could be related to the ability to coordinate the alkyne to the allyl-Pd intermediate $\mathbf{A}$. Thus, formation of $\mathbf{C}$ can only take place when a vacant coordination site is available. Therefore, dissociation of $\mathrm{PCy}_{3}$ is necessary for this reaction to occur. In the presence of a highly stronger coordinating IMes, the alkyne could not enter the coordination sphere, and the carbometallation could not take place. DFT calculations on model complexes support this idea. As expected, NHC ligand dissociation is far more difficult and may hamper the insertion of the alkyne into the allyl-Pd complex (Scheme 3).

The boronates we have prepared can be used for a number of synthetic applications. Thus, 3 ef was subjected to oxidation conditions to give the corresponding ketone 4 (Scheme 4). ${ }^{16}$

Some of the alkenylboronates (3ab, 3ef, and $\mathbf{3 p}$ ) were assayed in Suzuki reactions, affording the expected tetrasubstituted alkenes $\mathbf{5}$ in good yields, stereoselectively (Scheme 5) ${ }^{17}$ No isomerisation to conjugated dienes was observed. Compound $5 \mathbf{a b}$ could be more efficiently prepared by one-pot cyclisation of $\mathbf{1 a}$ (Table 2), followed by cross-coupling in $63 \%$ yield over two steps.

On the other hand, Suzuki coupling of allylboronates 2 led to the regioselective formation of the branched derivatives 6 (Scheme 6), albeit in lower yields. ${ }^{18,19}$

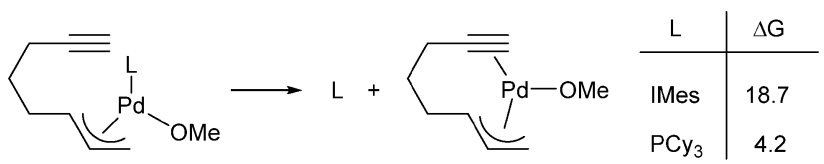

Scheme 3 Calculated $\Delta G$ of dissociation (kcal mol ${ }^{-1}$ ). M06/6-31G(d) (C,N,O,H) LANL2DZ (Pd), PCM (xylenes mixture).
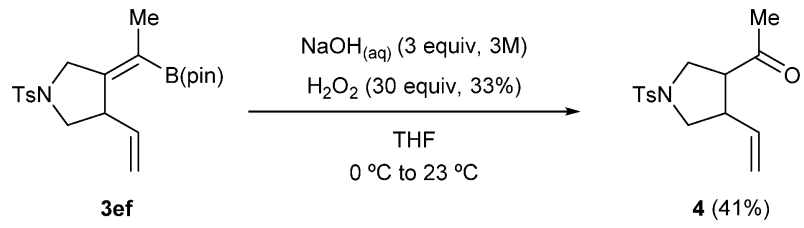

Scheme 4 Oxidation of an alkylboronate.

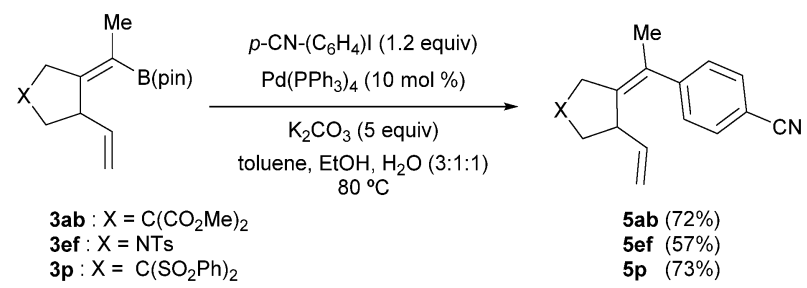

Scheme 5 Suzuki-Miyaura cross-coupling of alkenylboronates.
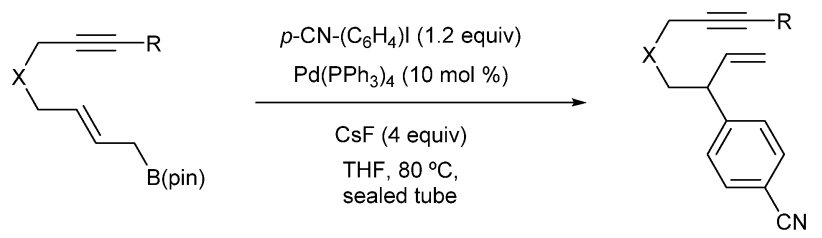

2ab : $\mathrm{X}=\mathrm{C}\left(\mathrm{CO}_{2} \mathrm{Me}\right)_{2}, \mathrm{R}=\mathrm{Me}$

2cd: $\mathrm{X}=\mathrm{C}\left(\mathrm{CO}_{2} \mathrm{Me}\right)_{2}, \mathrm{R}=\mathrm{Ph}$

2ef : $X=N T s, R=M e$

$6 \mathrm{ab}(52 \%)$

6cd $(51 \%)$

6ef $(33 \%)$

Scheme 6 Regioselective Suzuki coupling of allylboronates.

The resulting compounds are 1,7-enynes, which have proved to be valuable synthetic intermediates. ${ }^{20}$ Crystal structure of 6ef can be found in the ESI. $\dagger$ The observed regioselectivity is similar to that observed for related coupling reactions of allylboronates obtained by Pd-catalyzed cyclisation of dienynes. Buchwald has recently reported control on the regioselectivity of similar reactions by subtle differences in the ligands used. ${ }^{21}$

Finally, we would like to illustrate a potential interesting application of allylboronates. Terminal alkyne 7 was prepared by a different route, involving propargylation of allylboronate derived from malonate. Au-catalyzed cycloisomerisation afforded a mixture of two new isomeric allylboronates, which could not be separated. The observed mixture was expected, according to the previous work by Hall. ${ }^{22}$ Regioselectivity is dependent on the reaction temperature. The high reactivity of cationic Au complexes in these processes allows the lowering of temperature to $-78{ }^{\circ} \mathrm{C}$, which favours formation of the sixmembered derivative $(\mathbf{8}: \mathbf{9}, 1: 3$, Scheme 7$)$. Useful reactions for

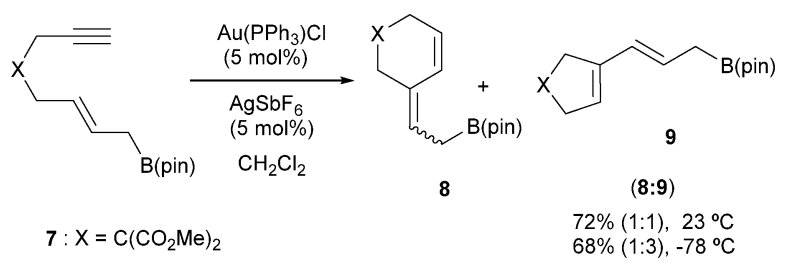

Scheme 7 Au-catalyzed cycloisomerisation reaction. 
the formation of allylboronates are expected to be developed using this approach. ${ }^{23}$

In summary, a borylative cyclisation leading to alkenylboronates has been developed. An appropriate choice of ligands allows formation of allylboronates. Synthetic utility of these compounds has been illustrated, suggesting potential future applications.

This work was supported by the MICINN (CTQ2010-15927) and the CAM (Project AVANCAT). We thank the UAM for a fellowship to R. L.-D., and the CCC-UAM for computation time.

\section{Notes and references}

1 D. G. Hall and H. Lachance, Allylboration of Carbonyl Compounds, Wiley, Hoboken, New Jersey, 2012; D. G. Hall, Boronic Acids, WileyVCH, Weinheim, Germany, 2005.

2 E. Jiménez-Núñez and A. M. Echavarren, Chem. Rev., 2008, 108, 3326; V. Michelet, P. Y. Toullec and J.-P. Genet, Angew. Chem., Int. Ed., 2008, 47, 4268; L. Zhang, J. Sun and S. A. Kozmin, Adv. Synth. Catal., 2006, 348, 2271; C. Aubert, O. Buisine and M. Malacria, Chem. Rev., 2002, 102, 813; G. C. Lloyd-Jones, Org. Biomol. Chem., 2003, 1, 215; A. M. Echavarren and C. Nevado, Chem. Soc. Rev., 2004, 33, 431.

3 S. T. Diver and A. J. Giessert, Chem. Rev., 2004, 104, 1317; B. Schmidt, Angew. Chem., Int. Ed., 2003, 42, 4996.

4 C. Bruneau, Angew. Chem., Int. Ed., 2005, 44, 2328; M. P. Muñoz, M. Méndez, C. Nevado, D. J. Cárdenas and A. M. Echavarren, Synthesis, 2003, 2898.

5 J. Marco-Martínez, V. López-Carrillo, E. Buñuel, R. Simancas and D. J. Cárdenas, J. Am. Chem. Soc., 2007, 129, 1874.

6 J. Marco-Martínez, E. Buñuel, R. Muñoz-Rodríguez and D. J. Cárdenas, Org. Lett., 2008, 10, 3619; J. Marco-Martínez, E. Buñuel, R. López-Durán and D. J. Cárdenas, Chem. - Eur. J., 2011, 17, 2734.

7 Enallenes and allenynes: V. Pardo-Rodríguez, J. Marco-Martínez, E. Buñuel and D. J. Cárdenas, Org. Lett., 2009, 11, 4548.

8 Borylative cyclisation of 1,7-enynes: V. Pardo-Rodríguez, E. Buñuel, D. Collado-Sanz and D. J. Cárdenas, Chem. Commun., 2012, 48, 10517.

9 Dienynes: R. López-Durán, A. Martos-Redruejo, E. Buñuel, V. PardoRodríguez and D. J. Cárdenas, Chem. Commun., 2013, 49, 10691.

10 Y. Deng, T. Bartholomeyzik and J. E. Bäckvall, Angew. Chem., Int. Ed., 2013, 52, 6283; A. K. A. Persson, T. Jiang, M. T. Johnson and J. E. Bäckvall, Angew. Chem., Int. Ed., 2011, 50, 6155.
11 Synthetic application of borylative cyclisations: A. M. Camelio, T. Barton, F. Guo, T. Shaw and D. Siegel, Org. Lett., 2011, 13, 1517.

12 Allylboronates from allylic acetates: P. Zhang, I. A. Roundtree and J. P. Morken, Org. Lett., 2012, 14, 1416. Seminal work: T. Ishiyama, T. Ahido and N. Miyaura, Tetrahedron Lett., 1996, 37, 6889.

13 Related Pd-catalyzed cascade reactions: C. H. Oh, H. R. Sung, S. J. Parks and K. H. Ahn, J. Org. Chem., 2002, 67, 7155; C.-W. Lee, K. S. Oh, K. S. Kim and K. H. Ahn, Org. Lett., 2000, 2, 1213; C. H. Oh and Y. M. Lim, Tetrahedron Lett., 2003, 44, 267.

14 Pd-NHC in cross-couplings: J. Nasielski, N. Hadei, G. Achonduh, E. A. B. Kantchev, C. J. O'Brien, A. Lough and M. G. Organ, Chem. - Eur. J., 2010, 16, 10844; N. Marion and S. P. Nolan, Acc. Chem. Res., 2008, 41, 1440; S. Roy and H. Plenio, Adv. Synth. Catal., 2010, 352, 1014.

15 Trialkylphosphines-Pd in couplings, leading references: I. D. Hills, M. R. Netherton and G. C. Fu, Angew. Chem., Int. Ed., 2003, 42, 5749; M. Iwasaki, S. Hayashi, K. Hirano, H. Yorimitsu and K. Oshima, J. Am. Chem. Soc., 2007, 129, 4463; C. A. Fleckenstein and H. Plenio, Chem. Soc. Rev., 2010, 39, 694; R. B. Bedford, M. B. Hursthouse, M. E. Light and E. J. M. Scordia, Dalton Trans., 2004, 3864.

16 H. R. Snyder, J. A. Kuck and J. R. Johnson, J. Am. Chem. Soc., 1938, 60, 105.

17 H. Zhou and C. Moberg, J. Am. Chem. Soc., 2012, 134, 15992.

18 L. I. Pilkington and D. Barker, J. Org. Chem., 2012, 77, 8156.

19 Allyl-aryl Suzuki coupling: J. L. Farmer, H. N. Hunter and M. G. Organ, J. Am. Chem. Soc., 2013, 134, 17470; S. Sebelius, V. J. Olsson, O. A. Wallner and K. J. Szabó, J. Am. Chem. Soc., 2006, 128, 8150; Y. Yamamoto, S. Takada and N. Miyaura, Organometallics, 2009, 28, 152.

20 Reviews containing examples of cyclisation of 1,7-enynes: O. Geis and H. Schmalz, Angew. Chem., Int. Ed., 1998, 37, 911; J. BlancoUrgoiti, L. Añorbe, L. Pérez-Serrano, G. Domínguez and J. PérezCastells, Chem. Soc. Rev., 2004, 33, 32; M. Rodríguez-Rivero, J. Adrio and J. C. Carretero, Eur. J. Org. Chem., 2002, 2881; S. T. Ingate and J. L. Marco-Contelles, Org. Prep. Proced. Int., 1998, 30, 123.

21 Y. Yang and S. L. Buchwald, J. Am. Chem. Soc., 2013, 135, 10642; S. Sebelius, V. J. Olsson, O. A. Wallner and K. J. Szabó, J. Am. Chem. Soc., 2006, 128, 8150 .

22 Alkenylboronates containing alkynes afford products with the opposite endo/exo regioselectivity, affording 6-member rings: J. C.-H. Lee and D. G. Hall, Tetrahedron Lett., 2011, 52, 321.

23 C. Nieto-Oberhuber, M. P. Muñoz, E. Buñuel, C. Nevado, D. J. Cárdenas and A. M. Echavarren, Angew. Chem., Int. Ed., 2004, 43, 2402 . 